\title{
Le texte dans l'apprentissage de la lecture en cours préparatoire : analyse et description
}

\author{
Véronique Quanquin \\ Projet AMICAL, Laboratoire de Recherche sur le Langage, \\ Université Blaise Pascal, Clermont-Ferrand 2 \\ quanquin@1rl.univ-bpclermont.fr
}

\section{Introduction}

Le développement des nouvelles technologies dans le domaine de l'apprentissage, outils spécifiques, STI, EIAH, ou encore enseignement en ligne (e-learning), l'ensemble des problématiques abordées ainsi que la nécessaire pluridisciplinarité des équipes ont ouvert un important champ de questionnement dans le domaine de la didactique.

C'est dans le cadre d'un projet de Système Tuteur Intelligent, le projet AMICAL (Architecture Multiagent Interactive Compagnon pour l'Apprentissage de la Lecture), que nous allons aborder le problème de la didactique en langue maternelle. Le contexte de l'étude porte sur un aspect essentiel de l'apprentissage de la langue, l'enseignement et l'apprentissage initial de la lecture en cours préparatoire. Une présentation générale du projet sera proposée afin de situer et développer de manière compréhensible l'une des décisions d'enseignement qui doit être prise par rapport au domaine, celle du choix du texte qui sera support et objet d'apprentissage.

Organiser l'apprentissage dans un environnement informatique consiste à créer des situations et à spécifier des problèmes qui permettront à l'apprenant d'acquérir les connaissances visées (Tchounikine in Grandbastien et Labat, 2006). Concernant l'apprentissage de la lecture, le texte a un rôle central à jouer : il permet les apprentissages nécessaires à la mise en place des deux composantes essentielles de la lecture, à savoir le décodage et la compréhension. L'analyse du texte est faite dans cette perspective.

Nous présentons dans un premier temps, le contexte de la recherche, à la fois par rapport au projet dans lequel la décision du choix du texte s'insère et par rapport à l'apprentissage de la lecture puis nous analysons le texte dans deux de ses rôles, en tant que support et en tant qu'objet de l'apprentissage de la lecture.

\section{Présentation générale de la recherche}

\subsection{Cadre de la recherche}

La recherche présentée dans cet article s'inscrit dans un projet de recherche universitaire pluridisciplinaire intitulé AMICAL. Ce projet de recherche a deux objectifs. Il s'agit tout d'abord d'associer les travaux de recherche actuels sur les différents domaines concernés -apprentissage de la lecture, didactique et pédagogie, sciences cognitives, linguistique- aux avancées des technologies de l'information et la communication, pour d'autre part utiliser les possibilités offertes par les environnements informatiques et élaborer un système informatique répondant de manière individualisée aux besoins de l'apprenant.

Le domaine d'application choisi est l'enseignement-apprentissage de la lecture en situation initiale, c'està-dire en début de cours préparatoire, pour des apprenants ne présentant pas de déficit cognitif particulier. La recherche se fait en équipe pluridisciplinaire intégrant des chercheurs dans tous les domaines 
concernés ainsi qu'en informatique; de plus un professeur des écoles a toujours été intégré à l'équipe et ce dès le début du projet.

L'outil est construit pour être intégré dans une classe, et constituer une aide à l'enseignement et à l'apprentissage de le lecture. Il est donc autonome, et complémentaire du travail effectué en classe.

Son autonomie est possible parce qu'il s'agit d'un système informatique à bases de connaissances, constitué d'agents collaborant aux différentes prises de décision d'enseignement pour un apprenant donné. Les prises de décision font partie d'un cycle fonctionnel d'enseignement (figure 1) et sont successives et interdépendantes ; les cycles d'enseignement se succèdent et ont chacun comme point de départ la représentation de l'élève qui a été construite par le système informatique et est mise à jour après chaque activité de l'apprenant. Ainsi l'enseignement est-il individualisé, c'est-à-dire construit, à chaque moment de son apprentissage, pour un apprenant particulier.

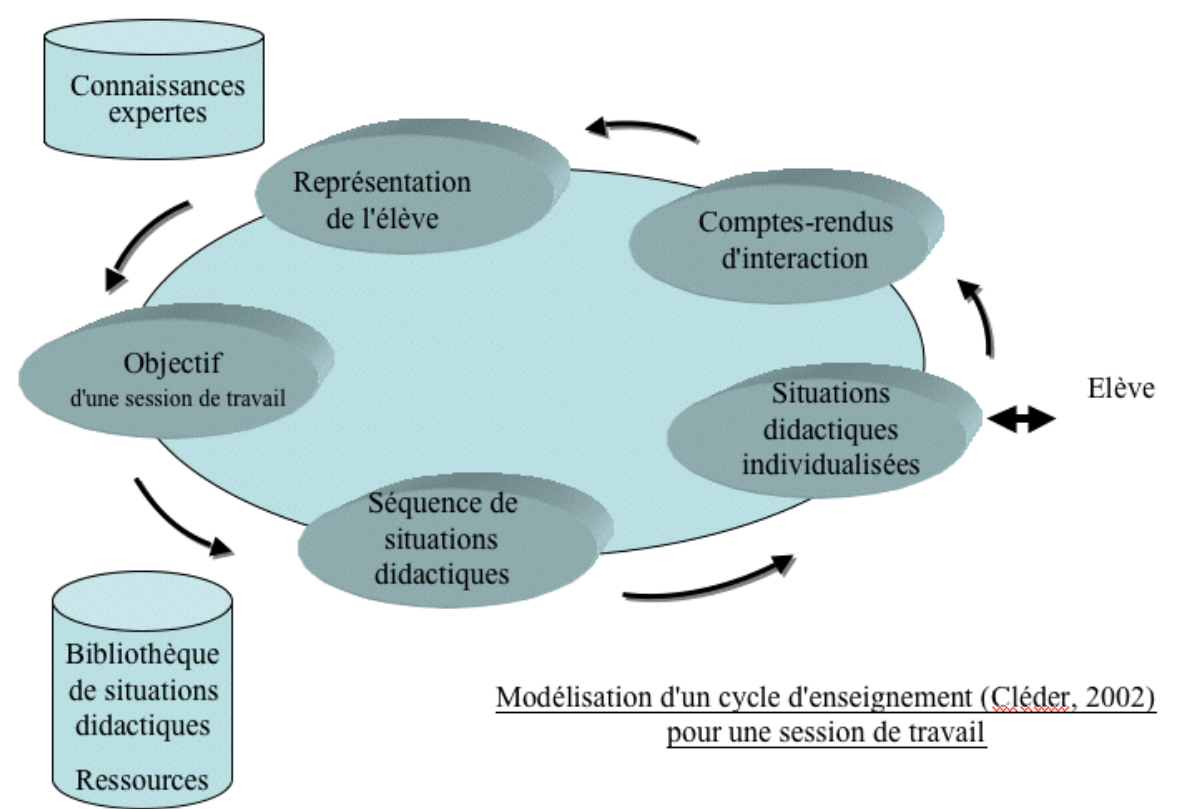

Figure 1 : Le cycle fonctionnel d'AMICAL

La première décision, présentée dans le schéma ci-dessus, concerne la construction de l'objectif d'enseignement-apprentissage. Elle est construite à partir des connaissances intégrées à la représentation de l'apprenant, en particulier d'un état des savoirs et savoir-faire de l'apprenant, mais aussi à partir des bases de connaissances spécialisées. Sur la base de cet objectif construit pour un apprenant donné, le système, par l'intermédiaire d'agents spécialisés, recherche dans une bibliothèque d'activités, les activités qui peuvent permettre d'atteindre cet objectif, il construit donc une séquence d'activités susceptibles de répondre à l'objectif défini. A ce stade des prises de décisions, les activités sont des « coquilles vides », elles ne sont pas instanciées par des valeurs particulières, comme par exemple le texte support de l'apprentissage, ou les mots qui seront à reconnaître dans le texte. Par conséquent, l'étape suivante est celle de l'instanciation des activités. Le résultat de ces trois premières étapes est la construction d'une séquence d'activités individualisées, qui doivent permettre à un apprenant particulier d'atteindre l'objectif d'apprentissage qui a été construit pour lui, étant donné son état de savoir. Suit l'exécution, par l'apprenant, de la séquence d'activités ; parallèlement, le système informatique récupère des informations, comme les réponses justes ou fausses, ou encore les essais avant validation, et construit le compte-rendu de la session de travail. Ce compte-rendu est ensuite interprété puis intégré à la représentation de l'élève afin de la mettre à jour. 
Ce cycle fonctionnel d'enseignement est constitué d'une succession de prises de décision, élaborées chacune à partir de différents ensembles de connaissances qui sont mis en relation avec la représentation de l'apprenant. L'enseignement est donc individualisé à différents niveaux : l'objectif d'enseignement, les activités et la séquence de ces activités, ainsi que les objets-supports de connaissance. Les ensembles de connaissances sont liés au domaine d'application, l'enseignement et l'apprentissage de la lecture, mais aussi à la didactique et à la pédagogie, et enfin à un domaine contribuant, la linguistique, parce que le savoir-faire lecture s'applique à un objet linguistique, le texte (Quanquin et Chambreuil, 2006).

La décision du choix du texte qui nous occupe est une micro-décision impliquée dans l'étape d'instanciation des activités. En effet, nous avons choisi de placer le texte au cœur de l'apprentissage de la lecture comme support et objet d'apprentissage, puisque apprendre à lire c'est apprendre à construire du sens. Le système doit donc choisir le texte qui conviendra le mieux au moment de l'apprentissage et à l'élève concerné, et sera support des activités de la séquence construite pour cet apprenant. Avant de présenter l'analyse de cette décision, il faut s'intéresser au domaine d'application, l'apprentissage de la lecture, afin de comprendre le rôle du texte dans cet apprentissage.

\subsection{Le domaine d'application : l'apprentissage de la lecture}

Les recherches sur l'apprentissage de la lecture se font autour de deux dimensions essentielles : l'identification de mots et la compréhension. La première composante qui comprend des points comme la compréhension du fonctionnement de l'écrit, la relation à l'oral, le principe alphabétique, le décodage, a donné lieu à de nombreuses études en psychologie cognitive notamment (voir synthèse dans Ecalle et Magnan, 2002), en didactique (Nonnon et Goigoux, dir., 2007 ; Giasson, 1990, 1995) mais aussi sous un angle plus linguistique (Colé et Sprenger-Charolles, 2003). En revanche, l'étude de la compréhension est moins abordée. Elle est plus souvent étudiée chez des lecteurs effectifs, déjà avancés. Or les apprenants de cours préparatoire doivent acquérir des compétences dans cette dimension. Des activités comme, par exemple, les questions de compréhension posées après un texte lu, sont pratiquées en classe, elles permettent de vérifier si le texte est compris. Mais peu voire pas d'activités existent pour apprendre à comprendre. De plus les études sur l'acquisition des stratégies de compréhension concernent des lecteurs sachant lire, et montrent l'intérêt de pratiques comme le résumé par exemple (Bianco et al in Gentaz et Dessus, 2004) mais qui sont des pratiques inexploitables avec des apprenants non lecteurs.

Nous avons choisi dans le projet AMICAL, de placer l'apprenant, dès le début de son apprentissage, dans une situation de lecture "réelle ", c'est-à-dire face à un texte. Le texte, dans cette situation, a alors trois rôles. Il est tout d'abord support de l'apprentissage de la lecture, il est donc porteur de la connaissance à acquérir : on pourra à partir de lui, faire acquérir le principe alphabétique, le fonctionnement de l'écrit ou encore les stratégies d'identification de mots et en particulier les relations phonèmes/graphèmes. Il est aussi objet d'apprentissage, il faut alors étudier des particularités textuelles comme le type, le genre ou les reprises anaphoriques et leurs rôles dans la construction du sens. Enfin, il peut faciliter l'apprentissage si l'on prend en compte son niveau de difficulté ou son thème. Ce sont ces trois rôles qui vont déterminer l'analyse du texte pour la prise de décision de son choix.

\subsection{Modélisation de la décision du choix du texte}

Le problème qui se pose est donc de choisir le texte qui instanciera en partie la séquence d'activités, pour un apprenant donné, sachant que le texte doit jouer ces trois rôles dans l'apprentissage de la lecture : support de connaissances, objet de connaissances et élément facilitateur. Que ce soit dans un contexte d'apprentissage de la lecture en classe, ou dans le contexte d'AMICAL, le texte est présenté au début de la séquence de travail, il doit tout d'abord être compris par l'apprenant, car on ne peut pas baser des apprentissages sur un support non maîtrisé. Il fait donc l'objet, après sa lecture par le système, d'une activité de vérification de compréhension et de mémorisation des informations. Puis, dans les activités qui suivent, il sera repris pour servir de support aux différents apprentissages. Cette décision du choix du texte se pose également pour un enseignant; ainsi, avant d'exposer la modélisation du choix du texte dans AMICAL, nous allons évoquer rapidement cette décision dans un contexte classe. 


\subsubsection{Le choix du texte : observations}

Les textes utilisés pour l'apprentissage de la lecture en classe sont soit des textes de méthodes de lecture soit des écrits choisis par l'enseignant. Dans le cas des méthodes, on observe que les textes sont écrits par les auteurs de la méthode et peuvent l'être pour permettre un apprentissage spécifique. Certaines méthodes comme Ribambelle font appel à des auteurs de littérature jeunesse pour écrire des textes destinés à l'apprentissage de la lecture. C'est le cas du texte "Quel bazar chez Zoé » utilisé comme exemple dans cet article. Ce qui est intéressant à noter dans ce cas, c'est la double compétence mise en œuvre, pas nécessairement par la même personne, d'auteur et de spécialiste de l'apprentissage de la lecture. Lorsque les enseignants choisissent eux-mêmes leurs textes, ils utilisent des textes que l'on peut qualifier d'authentiques : albums, poèmes, documentaires, textes prescriptifs (recette, règle du jeu...), ou « utilitaires» (menu, table des matières...). Ils utilisent aussi des textes écrits par les élèves soit de leur classe, soit d'autres classes, dans le cas d'une correspondance interclasse; enfin les textes peuvent être écrits par l'enseignant lui-même à partir ou non de propositions d'apprenants (Piquée et Sensevy, 2007).

L'observation de méthodes et la collaboration avec des professeurs des écoles montrent que les critères de choix mis en œuvre sont bien souvent : la longueur du texte et des phrases, la possibilité de travailler des relations phonèmes/graphèmes particulières, le thème. La syntaxe comme le lexique sont bien souvent évoqués en termes de difficulté ou facilité, la répétition, les connecteurs, les types de textes peuvent constituer des critères de choix ; l'intertextualité l'est rarement, c'est un véritable critère d'écriture des textes pour la méthode Croquepages qui promène la souris Quenotte dans les pages de «Hansel et Gretel » ou du « Petit Chaperon Rouge ».

Le questionnaire que Piquée et Sensevy ont transmis aux enseignants et concernant leurs pratiques en lecture, spécifie trois critères de choix de texte supplémentaires: "l'absence de problèmes de compréhension majeurs », "l'intérêt propre du texte », "la présence dans le texte d'un ou plusieurs problème(s) de compréhension que l'enseignant souhaite aborder » (ibid, 247).

\subsubsection{Le choix du texte dans AMICAL}

Dans le logiciel AMICAL, cette décision se base sur d'autres éléments. Le logiciel aura une banque de textes disponibles, textes stockés et non ordonnés en progression, et c'est de cette banque de textes que devra être extrait le texte qui convient. Il faut donc modéliser cette décision dans le contexte présenté. Le choix du texte peut se faire à partir de l'objectif : s'il est indiqué dans l'objectif que l'apprenant doit travailler le graphème 'ph', il faut que le texte contienne des mots qui présentent ce graphème. C'est la première étape de la décision du choix du texte qui consiste à faire correspondre les besoins et contraintes provenant de l'objectif, donc en lien avec l'apprentissage de la lecture, avec des connaissances spécifiques présentes dans le texte. Dans un deuxième temps, il est intéressant de profiter des particularités des textes potentiels et d'en faire des opportunités. Par exemple, si un des textes présente une série lexicale sur les instruments de l'orchestre qui peut être intéressante à étudier, alors le texte sera sélectionné pour cette particularité. Le choix du texte se fait donc en deux étapes successives : tout d'abord la sélection des textes permettant d'atteindre l'objectif, ensuite le choix, dans l'ensemble des textes possibles, d'un texte particulier en raison des opportunités qu'il présente.

Cette modélisation succinctement évoquée ici, suppose une démarche particulière et un outil indispensable. La démarche suivie est totalement différente de ce qui est généralement présenté dans les livres destinés aux enseignants ou dans les instructions officielles : nous ne caractérisons pas le texte en critères, mais à partir d'une analyse fine des besoins d'enseignement pour cet apprentissage individualisé, nous observons si les textes permettent ces apprentissages.

Un outil est indispensable à la réalisation de cette décision, il s'agit de la description du texte. En effet, que les textes supports soient écrits pour l'apprentissage de la lecture ou, comme dans le cas d'AMICAL, que ce soient des textes d'albums non conçus pour être utilisés dans ce contexte, la description a un rôle important à jouer. En effet, il faut rendre « utilisables », dans ce contexte, les textes d'album, autrement dit il faut analyser puis décrire ce qui peut être utilisé. Concernant les textes écrits pour l'apprentissage de 
la lecture comme «Quel bazar chez Zoé », leur analyse fine construit une connaissance plus approfondie du texte. L'outil sur lequel nous travaillons est plus qu'une description, il s'agit d'une description-analyse qui constitue l'outil central de la décision du choix du texte. Elle se présente sous deux états : sous forme de grille non instanciée comprenant tous les éléments intéressants à considérer étant donné le rôle du texte et permettant de décrire n'importe quel texte ; puis pour chacun des textes, sous forme de description propre, la grille est alors instanciée par les valeurs propres au texte décrit.

Dans la suite de l'article, c'est la grille non instanciée qui va être présentée, ainsi que les problèmes et questions qui se posent pour son élaboration et qui renvoient à deux pratiques didactiques différentes. Cette présentation sera exemplifiée par deux textes, l'un, «Quel bazar chez Zoé » (annexe 1), a été écrit par un auteur de littérature jeunesse pour la méthode Ribambelle, l'autre « Musique » (annexe 2), est un album qui peut devenir support d'apprentissage. La grille ne constitue cependant pas le seul outil nécessaire, d'autres structures de connaissances ont un rôle important à jouer dans le choix du texte ; elles seront aussi évoquées. Deux des rôles du texte dans l'apprentissage seront examinés successivement et permettront de s'intéresser aux savoirs théoriques de référence, ici en particulier la linguistique, par rapport à la transposition didactique (3ème partie) et par rapport à une reformulation (4ème partie). Notons enfin qu'il ne sera pas fait mention, dans cet article, des illustrations présentes dans les albums, notre propos étant centré sur la linguistique et le texte. La question des illustrations est cependant posée dans le logiciel AMICAL, et ce à plusieurs moments : une illustration peut être présente avant le texte ou à côté du texte au moment de sa présentation, les questions qui se posent alors sont en particulier celles de la redondance et/ou de la complémentarité par rapport à l'information; une autre utilisation de l'illustration est étudiée, c'est lorsque celle-ci est utilisée en tant qu'item de réponse à une question de compréhension par exemple.

\section{Analyse du texte en tant que support de l'apprentissage}

La description est un ensemble de connaissances, identifiées, formulées et organisées par rapport à une utilisation spécifique, ici la première dimension de l'apprentissage de la lecture, l'identification de mots écrits. C'est une structure de connaissances.

La méthode qui a permis d'élaborer cette grille a consisté à faire tout d'abord l'inventaire des savoirs linguistiques sur les objets centraux dans cette première dimension de l'apprentissage, la lettre et le mot. Concernant la lettre, les ensembles de lettres, phonèmes, graphèmes sont connus, les relations entre ces trois ensembles aussi, toutes les connaissances sont donc répertoriées (voir les travaux de N. Catach en particulier, ou ceux de J.-P. Jaffré sur l'orthographe). Dans un deuxième temps, ces savoirs linguistiques ont été analysés par rapport à l'apprentissage de la lecture et en particulier par rapport à ce qui doit être acquis pour développer ce savoir-faire. C'est cette confrontation qui a permis de sélectionner des éléments dans les savoirs linguistiques, de les exprimer et de les structurer dans cette grille ainsi que dans des structures de connaissances complémentaires. Deux exemples sur l'objet « lettre » vont être développés afin d'illustrer ces questions.

Si l'on considère que chaque texte contient tous ou presque tous les graphèmes et/ou les phonèmes français, il n'est pas intéressant ni pertinent d'intégrer à la description du texte des listes de mots contenant ces graphèmes et phonèmes. Ceci est d'autant plus vrai que l'informatique permet de répondre directement à des requêtes comme "sélectionner tous les textes contenant le graphème 'ph' ». Dans ce cas particulier, si l'objectif est de faire acquérir le graphème 'ph', le système informatique fera alors une fouille de texte et identifiera les textes contenant des mots présentant le graphème recherché. Ainsi les textes présentant ce graphème seront identifiés, et pour chacun, la liste des mots le contenant sera construite. Le savoir linguistique est donc présent, non pas pour constituer la description du texte, mais pour construire dynamiquement des ensembles de connaissances requises pour l'apprentissage comme les listes des phonèmes, des graphèmes, ainsi que les contextes spécifiques, par exemple « tout 'n' qui suit une voyelle ne forme pas obligatoirement une nasale ».

D'autres ensembles structurés de connaissances sont indispensables, non plus constitués pour faire de la récupération automatique de données, mais par rapport à des besoins liés à l'apprentissage de la lecture. 
Ce sont par exemple des ensembles présentant les graphèmes ordonnés en termes de fréquence dans la langue française, ou l'ensemble des graphèmes transcrivant un phonème. Ces ensembles permettront au système de faire des choix d'unités à acquérir : quel graphème faire acquérir en priorité étant donné sa fréquence, donc son degré d'utilité, et le savoir de l'apprenant à cet instant $t$ ? Ils ont été conçus à partir des unités répertoriées dans les savoirs linguistiques, et réorganisées par rapport à ce besoin spécifique d'enseignement. Ils permettent de construire dynamiquement une progression individualisée.

Le tableau suivant montre une structure de connaissances utilisées par le système informatique. Il s'agit d'un classement de tous les graphèmes, par rapport à un archigraphème ; ces graphèmes sont classés en termes de pourcentage d'utilisation dans la langue française. Ainsi, il est indispensable de faire acquérir aux apprenants le graphème ' $f$ ' car c'est celui qui est le plus utilisé. Cependant si les apprenants travaillent sur un texte contenant le mot «éléphant », il est tout à fait possible de leur faire acquérir cette deuxième graphie. Ces classements listent toutes les consonnes, voyelles et semi-voyelles de la langue française et permettent de faire des choix d'apprentissage qui construisent dynamiquement la progression.

\begin{tabular}{|l|l|l|l|l|l|}
\hline Archi graphèmes & Phonèmes & $\begin{array}{l}\text { Graphèmes } \\
\text { de base }\end{array}$ & $\begin{array}{l}\text { Pourcentage } \\
\text { d'utilisation }\end{array}$ & \multicolumn{2}{l|}{ Autres graphèmes } \\
\hline & & & $\begin{array}{l}\text { A introduire en } \\
\text { CP }\end{array}$ & $\begin{array}{l}\text { Ne pas introduire } \\
\text { en CP }\end{array}$ \\
\hline C & {$[\mathrm{k}]$} & $\begin{array}{l}\mathrm{c}+\mathrm{a}, \mathrm{o}, \mathrm{u} \\
\mathrm{c}+\mathrm{cons} \\
\mathrm{qu}\end{array}$ & $98 \%$ & $\mathrm{k}, \mathrm{q}$ (final), cc & cqu, ch, cch, ck \\
\hline F & f $]$ & $\mathrm{f}$ & $95 \%$ & $\mathrm{ff}, \mathrm{ph}$ & \\
\hline etc & & & & & \\
\hline
\end{tabular}

Figure 2 : Exemple de classement des graphèmes et phonèmes

Les connaissances autour de l'objet « lettre » sont donc structurées d'une part, pour choisir la connaissance à faire acquérir c'est-à-dire créer l'objectif d'apprentissage, d'autre part pour construire les listes de mots ayant certaines particularités. Ces aspects, même s'ils n'apparaissent pas dans la description du texte, sont indispensables pour le choisir. Ce qui en revanche sera intégré à la description du texte, ce sont des connaissances par rapport aux difficultés potentielles d'apprentissage. On sait que les apprenants peuvent avoir des difficultés en raison de la discrimination auditive. Par exemple les consonnes $\mathrm{R}$ ou $\mathrm{L}$ lorsqu'elles suivent une autre consonne, dans des digrammes comme «br » ou « bl », sont difficilement entendues et repérées par les apprenants ; ou bien la proximité phonétique des consonnes $\mathrm{T}$ ou $\mathrm{D}$, différentes d'un seul trait, la sonorité, peut entraîner des confusions (Colé et Sprenger-Charolles, 2003). D'autres difficultés comme des difficultés orthographiques sont également identifiées : par exemple, la lettre $\mathrm{M}$ remplaçant le $\mathrm{N}$ dans les nasales est justifiée par la présence des lettres $\mathrm{M}$, B ou $\mathrm{P}$ suivant la nasale.

Ces difficultés potentielles sont intéressantes à faire apparaître dans la description, car elles peuvent constituer un critère de choix du texte. Seront sélectionnés les textes présentant un ensemble d'occurrences qui permet de travailler l'une ou l'autre de ces difficultés. Il faut donc les identifier et les répertorier à partir du savoir théorique sur l'apprentissage de la lecture. La linguistique est aussi nécessaire à cette élaboration car elle permet de faire une description complète du problème (par exemple les traits phonétiques pour les consonnes T et D) ; elle permet aussi, par la mise en relation d'éléments, d'élaborer des structures de connaissances utiles dans les prises de décision. L'apport consiste à présenter des ensembles complets de connaissances répondant à des problèmes spécifiques d'apprentissage.

L'objet « lettre » décrit dans le texte «Quel bazar chez Zoé ! » (annexe 1) se présente ainsi, dans une expression non formalisée pour un système informatique : 


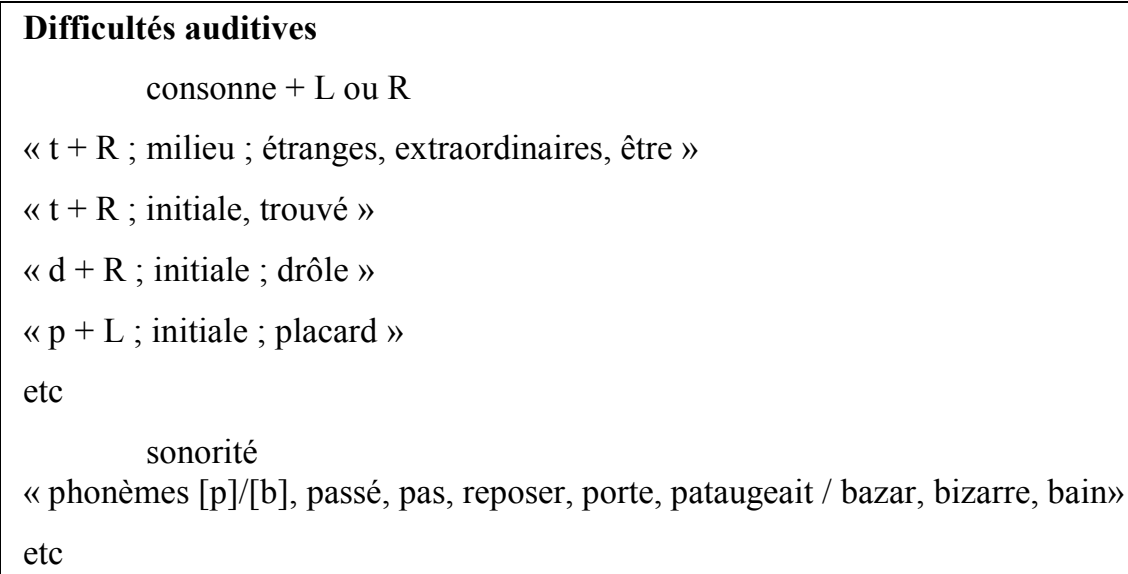

\section{Difficultés orthographiques}

nasales en $\mathrm{n}$ ou $\mathrm{m}$

«graphème 'en': en, attendre, vendredi, s'endormir, ennuyée, silence, sagement, silencieusement ; graphème 'em' : semblait, temps »

etc

1 lettre dans plusieurs graphèmes

« lettre a,

graphème ai, semaine, maison, extraordinaire, j'ai ;

graphème au, pataugeait ;

graphème eau, $\varnothing$;

graphème an, dans, étrange, dérangé ;

graphème am, Ø;

graphème ain, bain, main ;

graphème aim, $\varnothing$ »

etc

Figure 3 : Une partie de la description non formalisée de l'objet « lettre » pour le texte « Quel bazar chez Zoé »

Concernant l'objet « lettre », sa description théorique linguistique étant totale ou presque, il est possible de faire l'inventaire des connaissances le concernant. Il s'agit alors de réorganiser ces savoirs théoriques par rapport à une utilisation particulière, ici choisir un texte support d'apprentissage. La réorganisation de ces savoirs s'opère à partir des connaissances issues du domaine d'application, l'apprentissage de la lecture. Le travail en équipe pluridisciplinaire permet une collaboration étroite entre linguistes, spécialistes de l'apprentissage de la lecture et des sciences cognitives, avec la professeure des écoles associée. En effet, les propositions faites dans cet article ont fait l'objet de discussions avec l'enseignante qui collabore au projet; elles ont été réajustées suite à ces échanges. Signalons aussi qu'un travail de thèse est en cours sur la phrase qui permettra de décrire cet objet dans la grille. 
L'utilisation du savoir théorique dans cette première perspective constitue une transposition didactique. La collaboration chercheur/enseignant de terrain permet de concevoir, à partir du savoir théorique, les outils nécessaires à l'enseignement. La deuxième spécificité de ce travail est liée au contexte, en effet travailler pour construire un environnement informatique d'apprentissage oblige à une explicitation totale des connaissances mises en jeu pour les prises de décision.

\section{Analyse du texte en tant qu'objet de l'apprentissage}

Concernant la deuxième dimension de l'apprentissage de la lecture, la compréhension, le texte joue un rôle d'objet de l'apprentissage. L'idée n'est donc pas de savoir si le texte permet ou non tel apprentissage sur la lettre ou le mot, il s'agit plutôt d'identifier les éléments sur lesquels s'appuie le lecteur pour construire du sens. Les éléments linguistiques textuels sont donc à considérer par rapport aux stratégies de compréhension que le lecteur met en œuvre et que l'apprenti lecteur doit apprendre à mettre en œuvre.

La lecture des travaux linguistiques a permis de mettre en évidence les thématiques «classiques »de l'analyse du texte comme les séquences et types de textes, la progression thématique, la cohérence et la cohésion, les connecteurs et organisateurs textuels, le point de vue etc. La question qui se pose nécessairement après cet inventaire est celle de la globalité du texte. Différents travaux ont été utilisés, en particuliers ceux de J.-P. Bronckart (1996), E. Roulet et al. (2001), et J.-M. Adam (2006). Puis une analyse systématique des albums utilisés par les enseignants de cours préparatoire, ainsi que de méthodes de lecture ont permis de commencer à construire la grille de description-analyse du texte (Quanquin, 2000).

Pour construire cet outil, deux méthodologies sont utilisées. Soit la théorie existe, elle est donc utilisée pour décrire le texte en tant qu'objet de l'apprentissage, et pour construire la grille d'analyse. Ou bien, la particularité textuelle observée dans l'analyse des albums nécessite de convoquer différentes théories, et il faut dans ce cas, répertorier, observer dans les albums, mettre en relation ces résultats avec les éléments théoriques disponibles et construire une théorisation de cet aspect. Les deux parties ci-dessous illustrent ces deux possibilités.

Dans les deux cas, et c'est une particularité liée à l'objet texte, l'analyse et la description du texte permettent de choisir le texte en sachant ce qui dans le texte peut être acquis par l'apprenant et peut donc faire l'objet d'activités d'apprentissage de la compréhension. Elles permettent aussi d'analyser les erreurs de l'apprenant et de lui donner l'aide qui correspond à ses besoins.

\subsection{L'utilisation des connaissances linguistiques}

L'analyse du texte « Musique » montre qu'il est constitué de 6 micro-narrations composées chacune des 5 mêmes propositions narratives :

- situation initiale : une souris joue de la musique,

- complication : cela embête la souris de la chambre suivante,

- réaction : celle-ci se met en colère,

- résolution : la première souris doit aller jouer sur la terrasse,

- situation finale : la deuxième souris est débarrassée du bruit de sa colocataire.

Ces micro-narrations sont successives; elles sont enchassées dans une narration plus globale, avec une situation initiale présentant les sept souris musiciennes qui habitent dans la même maison, une situation finale dans laquelle on retrouve toutes les souris en train de jouer de la musique, et enfin une morale indiquant que cette musique «n'était pas mal du tout » et que les souris ont formé, ensemble, un orchestre qui réjouit les habitants du quartier. L'analyse théorique élaborée par J. M. Adam (1992) s'applique sans difficulté à ce texte. 
Par rapport à l'apprentissage de la lecture, la succession de micro-narrations peut permettre de mettre en place la stratégie d'anticipation en lecture. Il est possible, à partir de l'analyse du premier texte et de la mise en évidence de la structure narrative, de faire acquérir aux apprenants un savoir explicite sur ce type de texte ; signalons qu'explicite ne signifie pas une utilisation de la métalangue obligatoirement, l'explicitation peut se faire dans des termes connus de l'apprenant. Il s'agit alors de faire acquérir à l'apprenant des connaissances concernant ce type de texte. Ces connaissances constituent un des moyens d'imaginer la suite de n'importe quel texte de type narratif quelle que soit la complexité de sa structure, donc d'anticiper la suite du texte. L'anticipation peut concerner, comme dans « Musique », une micronarration complète, elle peut se faire aussi à un moment dans la narration («que va faire le personnage principal suite au problème qu'il rencontre? »). Elles permettent aussi de faire produire des histoires à l'apprenant. Enfin, cette connaissance comme toutes celles concernant le texte, une fois acquise, permet d'alléger la charge cognitive liée à la compréhension d'une histoire. La description et l'analyse linguistique sont ainsi traduites en stratégie de construction du sens à acquérir par l'apprenant.

Une autre particularité textuelle peut être analysée, il s'agit de la progression thématique. «Quel bazar chez Zoé » présente, dans la première phrase du texte, le lieu dans lequel va se dérouler l'action (la maison de Zoé), le temps, ce sera une semaine, et ce qui se passe: «des tas de choses étranges, extraordinaires ». Le lieu et le temps assurent dans le texte la permanence et l'apport d'information, en effet il y a évolution dans la semaine et dans la maison. C'est la "colonne vertébrale » du texte. Le « tas de choses étranges, extraordinaires » est un hyperthème explicite qui est développé, donc précisé, par les différents sous-thèmes qui lui sont liés. Chaque jour apporte un nouvel événement étrange, extraordinaire. Cette analyse permet de faire faire des inférences, en particulier à partir des aspects temps et lieu ; l'hyperthème permet de travailler avec l'apprenant sur un point sémantique, ici l'étrangeté des situations, caractère qui rassemble toutes les situations. L'analyse concernant le texte « Musique » est différente sur ce point. Il nous semble plus pertinent, plutôt que de parler d'hyperthème, d'utiliser les notions de partie/tout. En effet, les «tout» présentés dès le début du texte concernent la maison, les souris et la musique. Dans les micro-narrations suivantes, un élément est isolé de chacun de ces «tout» et mis en relation avec les autres. A la fin du texte, toutes les parties sont réunies, comme au début, mais dans une formulation totalement différente qui met en évidence l'évolution thématique dans le texte : la maison est évoquée par la terrasse, les souris par l'arc en ciel et le groupe, et la musique par l'orchestre et le groupe. On voit bien dans cette structure, le thème du texte qui consiste à montrer que l'ensemble est plus riche (musique agréable, bonheur de chaque souris) que l'accumulation d'individualités. Cette structure identifiée, qui permet des inférences par rapport aux micro-narrations successives et à la situation finale, peut aussi être utilisée comme support de production par les apprenants.

\subsection{L'analyse des répétitions}

Les deux textes qui exemplifient cet article présentent beaucoup de répétitions. Les reprises, répétitions, reformulations sont beaucoup étudiées en linguistique en particulier par rapport à l'acquisition du langage (Martinot, dir, 2000), et par rapport à leur rôle dans l'interaction verbale (Vion, 1992, 2000); ces actes de reformulation sont analysés et classés par rapport aux marqueurs qui les introduisent, en fonction aussi de leur rôle dans l'évolution du sens du texte ou du discours (Adam et Herman, 2000). Une observation systématique des répétitions (nous utilisons ce terme dans un sens global qui regroupe ce qui se répète à l'identique et ce qui est reformulé dans des termes différents) a été faite dans les albums pour enfants. L'objectif est de construire un cadre théorique concernant ces particularités textuelles, afin de les identifier dans les textes et de savoir quels peuvent être leurs rôles dans la compréhension d'un texte, ou l'apprentissage de la compréhension, et dans la production. Nous les avons donc répertoriées, classées et caractérisées à partir des théories linguistiques, de celles sur la compréhension et des savoirs concernant l'enseignement-apprentissage de la lecture. Nous allons présenter trois exemples des répétitions afin de montrer d'une part que leur analyse mobilise des théories différentes, et d'autre part que leur impact n'est pas de même nature dans l'apprentissage de la construction du sens d'un texte.

Un premier type de répétition, facilement perceptible, est la reprise d'une phrase entière : «C'est étrange, c'est bizarre, non mais quel bazar ! » ou bien «Va jouer sur la terrasse ». Ces phrases sont répétées dans 
chacun des micro-textes du début de l'album, eux-mêmes identiques en termes de déroulement, structures, voire lexique. Ces répétitions peuvent être analysées comme étant des marqueurs de séquence textuelle, par exemple « va jouer sur la terrasse » est un marqueur de fin de séquence; de plus elles jouent un rôle structurant «en induisant des relations d'équivalence » entre les séquences (Adam, 2006) et marquent le fait que les micro-textes, parce qu'ils sont identiques, forment un tout. Il est possible de considérer que cette répétition constitue un liage de signifiant. En termes de sens, ces répétitions accentuent l'effet d'accumulation. Il se passe tous les jours la même chose chez Zoé donc accumulation dans le temps, ou bien chaque souris réagit de la même façon à la musique de sa voisine, cette réaction répétée provoque la désertion de l'appartement et la constitution d'un groupe sur la terrasse. L'arrêt de la répétition marque, quant à lui, un changement, une rupture dans l'évolution de l'histoire.

Par rapport à l'apprentissage de la lecture, une répétition de ce type est un outil très intéressant. Tout d'abord, l'apprenti lecteur sera vite capable de la repérer et de la lire en autonomie. De plus, le fait que la phrase soit strictement identique montre au lecteur que l'identité des textes, qu'il peut infèrer s'il fait l'analyse des micro-textes, est bien réelle; la répétition rend l'identité explicite. Ainsi, pour chaque «hôte » qui s'installe dans sa maison, Zoé s'exclame sur la bizarrerie de la situation; cette phrase déclenche la ou les actions qui suivent. Il est alors possible de questionner l'apprenant avant la lecture sur ce qui peut se passer. Enfin, en production, on peut demander à l'apprenant d'inventer l'histoire de la souris suivante, ou d'un autre jour de la semaine, avec utilisation de la phrase répétée.

Le texte "Musique » présente un autre type de répétition beaucoup moins habituel. En effet, certaines phrases que l'on retrouve dans tous les micro-textes sont identiques d'un point de vue syntaxique, et différentes lexicalement. Ce sont, par exemple, les phrases comme «mais ça dérangeait la souris grise de la chambre rose » et «mais ça contrariait la souris orange de la chambre jaune ». On peut considérer que l'identité syntaxique permet aussi de structurer le texte. Mais ce qui est intéressant dans ce cas, c'est la richesse lexicale de la reformulation. L'identité de la structure syntaxique est donc doublée d'une reformulation de l'idée principale. Ces deux répétitions permettent de faire comprendre et acquérir, par l'apprenant, des mots ou expressions comme «irriter, emboucher son saxophone, tintamarre » qui sont peu utilisés par des enfants de 6 ans, voire inconnus. La répétition permet d'alléger la charge cognitive lors de la lecture, elle rend possible un vrai travail d'acquisition de vocabulaire à partir du texte, elle montre aussi à l'apprenant qu'il est possible de dire la même chose avec différents mots. Enfin, elle montre à l'apprenti lecteur qu'il est possible d'inférer le sens d'un mot inconnu à partir du contexte. En production, c'est un moyen de montrer comment enrichir, améliorer un texte.

Enfin, nous avons souligné la répétition du schéma narratif dans les premiers micro-textes de « Musique ». En ce qui concerne le texte «Quel bazar chez Zoé ! », les 5 premiers textes ne peuvent pas être analysés par rapport à leur structure narrative. Ils sont plutôt construits sur la base d'un schéma cognitif général du type résolution de problème ; en effet Zoé perçoit et identifie le problème (elle trouve un ours dans le placard qui a l'air fatigué), elle réfléchit ( «C'est étrange, c'est bizarre, non mais quel bazar!»), puis elle fait une action qui va résoudre le problème (elle ferme la porte de la chambre). Comme pour le texte « Musique », le fait de retrouver le même schéma dans tous les micro-textes, même s'il est d'une autre nature, permet d'anticiper. Mais l'intérêt se trouve aussi dans la possibilité d'utiliser cette connaissance par rapport à des problèmes rencontrés dans la «vraie vie ». La principale difficulté, que l'enseignant ne rencontrera pas avec le schéma narratif, est dans la transmission de ce savoir à l'apprenant en raison du caractère abstrait de ce schéma.

Cette typologie des répétitions est présente dans la grille de description du texte, accompagnée de règles de décision permettant au système AMICAL de faire des choix parmi ces possibilités.

La grille de description du texte présente donc des items concernant l'objet texte qui sont issus soit d'une reformulation faite à partir de différents référents théoriques, soit d'une transposition didactique. Les items qui sont présentés dans cette grille sont pertinents par rapport à l'enseignement-apprentissage de la lecture, mais ils doivent être mis en relation les uns avec les autres. En effet, la difficulté du lexique utilisé dans «Musique " est à mettre en relation avec toutes les répétitions de tous les types qui sont 
utilisées. Ainsi, un des moyens de mettre en évidence la globalité du texte, et donc sa complexité, est de mettre les différents éléments qui le constituent en relation.

Autre point important qui montre la souplesse de l'outil, toutes les rubriques présentes dans la grille générale ne sont pas nécessairement instanciées ; par exemple le type du texte «Quel bazar chez Zoé ! » ne sera pas décrit, car il est difficile à isoler et peu visible, il ne présente donc pas vraiment d'intérêt pour son exploitation en termes de stratégie de compréhension, en revanche c'est le schéma cognitif qui sera décrit. C'est l'inverse pour le deuxième texte.

\section{Conclusion}

La question du choix du texte a été posée dans un contexte particulier d'utilisation : le texte comme support et objet de l'apprentissage d'un savoir-faire complexe, dans une perspective d'individualisation c'est-à-dire d'adaptation à un apprenant spécifique, et enfin dans un environnement informatique d'aide à l'apprentissage. Toutes ces dimensions ont influé sur l'analyse de cette décision et sur la construction des ensembles de connaissances utilisés dans cette prise de décision.

La pluridisciplinarité constitue une particularité de cette recherche. Elle est effective à au moins trois niveaux. Tout d'abord dans la collaboration inter-discipline. Comme nous l'avons indiqué, la modélisation de la décision de choix du texte ainsi que la construction des outils nécessaires utilisent des référents théoriques différents comme entre autres les sciences cognitives, en particulier les études sur la compréhension, ou la linguistique. Ensuite, pour passer de l'expertise à la création d'outils informatiques d'aide à l'apprentissage, une compétence informatique est indispensable. Il s'agit alors de faire une transposition informatique (Tchounikine in Grandbastien et Labat, 2006) qui permet une représentation symbolique de la connaissance et la mise en œuvre de cette représentation dans l'environnement d'apprentissage. Cette transformation, qui a été évoquée et non traitée dans cet article, met au jour d'autres questions qui peuvent conduire à des interrogations théoriques et à la modification de l'expertise.

Ces deux types de collaboration, entre les sciences humaines d'une part, et avec l'informatique d'autre part, conduisent à une explicitation totale des connaissances en jeu et de leur organisation. Cette démarche est particulièrement intéressante dans le cas de l'enseignement-apprentissage d'un savoir-faire comme la lecture dont l'enseignement ne fait pas l'objet d'une méthode admise par tous; elle permet en effet de différencier les approches et aussi de modéliser la progression dans cet apprentissage. Elle est aussi pertinente pour une discipline comme la linguistique car elle conduit à poser les questions théoriques autrement. C'est ce que nous avons essayé de montrer avec l'analyse de la répétition. Des questions de recherche peuvent ainsi émerger de cette collaboration.

Enfin, la pluridisciplinarité est intéressante car elle conduit à une réelle collaboration terrain-théorie, en reformulant et réorganisant les savoirs théoriques par rapport à des réelles questions d'enseignement, en justifiant le plus possible par un savoir de référence, les choix et les analyses, en optimisant l'utilisation d'un outil particulier comme l'album. Dans le cas du choix du texte, celui-ci ne se fera plus intuitivement, mais à partir d'un savoir identifié. L'interaction terrain-théorie est réelle du fait de la constitution de l'équipe, ainsi chaque avancée théorique est discutée et modifiée en fonction de l'expertise de l'enseignant qui apporte aussi ses questionnements.

Nous avons montré que l'outil informatique nécessite une explicitation complète et fine des savoirs de références mis en jeu, des connaissances et des décisions. Cette expertise, parce qu'elle est totalement explicitée, peut être transmise à un enseignant: c'est un des intérêts majeurs de la recherche sur les environnements d'aide à l'apprentissage.

Le projet AMICAL est vaste, par conséquent, non encore réalisé dans sa totalité. Cependant une valorisation de cette recherche universitaire est en cours actuellement. En effet, un outil indépendant d'aide au choix du texte est en cours de réalisation, il sera destiné aux enseignants pour une utilisation autonome, hors logiciel d'aide à l'apprentissage de la lecture. Cet outil est construit à partir de la modélisation de la décision du choix du texte dans le cadre d'AMICAL. Il comprendra une base de textes analysés et aidera l'enseignant à faire le choix du texte pertinent au moyen de boîtes de dialogue, d'aides 
particulières et d'une navigation organisée. L'expertise présentée dans cet article est donc utilisée et, par cet outil partiel, transmise à l'enseignant.

\section{Références bibliographiques}

Adam, J. M. (2006). La linguistique textuelle. Introduction à l'analyse textuelle des discours. Paris : Armand Colin.

Adam, J. M. (1992). Les textes : types et prototypes. Récit, description, argumentation, explication et dialogue. Paris : Nathan-Université

Adam, J. M. Herman, T. (2000). Reformulation, répétition et style périodique dans l'appel du 18 juin 1940. Semen, 12.

Bronckart, J. P. (1996). Activité langagière, textes et discours. Pour un interactionnisme socio-discursif. Lausanne : Delachaux et Niestlé.

Cléder, C. (2002). Planification didactique et construction de l'objectif d'une session de travail individualisé : modélisation des connaissances et du raisonnement mis en jeu. Thèse de doctorat, Université Blaise Pascal, Clermont-Ferrand.

Colé, P. Sprenger-Charolles, L. (2003). Lecture et dyslexie, approche cognitive. Paris : Dunod.

Ecalle, J. Magnan, A. (2002). L'apprentissage de la lecture, fonctionnement et développement cognitifs. Paris : Armand Colin.

Gentaz, E. Dessus, P. (2004). Comprendre les apprentissages. Sciences cognitives et éducation. Paris : Dunod.

Giasson, J. (1990). La compréhension en lecture. Québec : Gaëtan Morin Editeur.

Giasson, J. (1995). La lecture, de la théorie à la pratique. Québec : Gaëtan Morin Editeur.

Grandbastien, M. Labat, J. M. (2006). Environnements informatiques pour l'apprentissage humain. Paris : Lavoisier.

Martinot, C., dir. (2000). Acquisition et reformulation. Langages, 140.

Nonnon, E. Goigoux, R., dir. (2007). Travail de l'enseignant, travail de l'élève dans l'apprentissage initial de la lecture. Repères, 36.

Piquée, C. Sensevy, G. (2007). Lecture au cours préparatoire: une analyse empirique de l'influence des choix pédagogiques et didactiques. Repères, 36, 231-254.

Quanquin, V. Chambreuil, A. (2006). Modélisation pour un environnement multimédia de l'apprentissage individualisé de la lecture. Psychologie Française, 51, 41-54.

Quanquin, V. (2000). Le choix du texte: problématique et application dans le cadre d'un environnement informatique d'aide à l'enseignement et à l'apprentissage de la lecture en cours préparatoire. Thèse de doctorat, Université Blaise Pascal, Clermont-Ferrand.

Roulet, E. Filliettaz, L. Grobet, A. (2001). Un modèle et un instrument d'analyse de l'organisation du discours. Bern : Peter Lang.

Vion, R. (1992, 2000). La communication verbale. Analyse des interactions. Paris : Hachette Supérieur.

\section{Annexes}

Annexe 1 : «Quel bazar chez Zoé ! » Dominique Falda

Cette semaine, dans ma maison, il s'est passé des tas de choses étranges, extraordinaires. C'est étrange, c'est bizarre, quel bazar!

Lundi, quelle drôle d'histoire, j'ai trouvé un ours velu dans mon placard. Il semblait fatigué. Il ne voulait pas être dérangé. Alors j'ai dit : «C'est étrange, c'est bizarre, non mais quel bazar ! » Et j'ai fermé la porte à clef pour qu'il puisse se reposer. 
Mardi, quelle drôle d'histoire, il y avait une girafe qui pataugeait dans mon bain. Elle semblait avoir soif. Alors j'ai dit : «C'est étrange, c'est bizarre, non mais quel bazar ! » Et je l'ai laissée dans la baignoire pour qu'elle puisse y boire.

Mercredi, quelle drôle de surprise, j'ai ouvert le réfrigérateur, un petit pingouin m'a saluée. Il semblait avoir trop chaud. Alors j'ai dit : «C'est étrange, c'est bizarre, non mais quel bazar ! » Et je l'ai déposé dans le congélateur pour qu'il puisse se rafraîchir, manger quelques glaces.

Jeudi, quelle drôle d'histoire, j'ai découvert dans mon jardin une princesse perchée sur une citrouille. Elle avait une pantoufle dans la main et semblait attendre quelqu'un. Alors j'ai dit : " C'est étrange, c'est bizarre, non mais quel bazar !» Et je lui ai offert un livre pour qu'elle puisse passer le temps.

Vendredi, quelle drôle de surprise, il y avait un montre vert caché sous mon lit. Il ne voulait pas sortir mais n'arrivait pas à s'endormir. Alors j'ai dit : «C'est étrange, c'est bizarre, non mais quel bazar ! » Et je lui ai prêté ma poupée préférée en lui souhaitant bonne nuit.

Et samedi, que s'est-il passé ? Samedi, il n'y a rien eu : pas d'ours fatigué, pas de girafe assoiffée, pas de pingouin en sueur, pas de princesse qui s'ennuie, pas de monstre insomniaque. Alors j'ai dit : " C'est étrange, c'est bizarre, pas de bazar. » Toute seule, je me suis ennuyée.

Dimanche, je les ai tous invités. L'ours est venu avec un oreiller, la girafe avec une serviette de bain, le pingouin avec des patins à glace, la princesse avec son prince charmant et le monstre vert avec ma poupée. Alors j’ai ciré : « Nous allons faire le bazar ! » Et on s'est bien amusés. Et puis j’ai dit : « Silence, nous allons regarder un film étrange et bizarre à la télévision. » Alors on s'est tous assis sur le canapé, sagement, silencieusement...

Et on s'est tous endormis !

Annexe 2 : « Musique » Claude Boujon

Sept souris habitaient la même maison. Elles avaient chacune une chambre et faisaient toutes de la musique.

Dans la chambre verte, la souris marron jouait de la batterie. Mais ça ne plaisait pas à la souris bleue qui logeait dans la chambre ocre. "Assez !" criait-elle en colère, "je ne m'entends pas moi-même. Va jouer sur la terrasse." Débarrassée du tonnerre de la batterie, la souris bleue jouait de la trompette.

Mais ça irritait la souris verte de la chambre blanche. "Tu me transperces les oreilles !", hurlait-elle. "Va jouer sur la terrasse." La trompette une fois partie, elle embouchait son saxophone.

Mais ça dérangeait la souris grise de la chambre rose. "Impossible de se concentrer avec un tel boucan!", gémissait-elle. "Va jouer sur la terrasse." Le saxophone disparaissait et la souris grise plaquait des accords sur sa guitare électrique.

Mais ça gênait la souris jaune de la chambre bleue. "Cesse ce tintamarre !" exigeait-elle, "tu me fais faire des fausses notes. Va jouer sur la terrasse." La guitare électrique s'en allait et la souris jaune se mettait à son violon.

Mais ça contrariait la souris orange de la chambre jaune. "Arrête ton crin-crin qui me casse la tête !" ordonnait-elle, "et va jouer sur la terrasse." Quand le violon n'était plus là, elle faisait des trilles sur sa flûte.

Mais ça indignait la souris noire qui habitait la dernière chambre de l'appartement. "Comment je chante, moi, avec tout ce vacarme ?" se plaignait-elle. "Va jouer sur la terrasse."

Après la sortie de la flûte, elle n'avait plus envie de chanter, car qui aurait-elle dérangé ? Elle rejoignait tout simplement les autres sur la terrasse...

... où chacune interprétait son petit air. 
Didactique et enseignement, français langue maternelle, français langue seconde DOI $10.1051 / \mathrm{cmlf0} 113$

Eh bien, cette musique n'était pas mal du tout. Elle mettait en joie les habitants du quartier. Ils avaient baptisé l'orchestre des sept souris : LE FANTASTIQUE ARC-EN-CIEL TERRASSE GROUPE. 